\title{
Measurement of Higgs boson couplings and differential distributions at CMS
}

\section{Matthias Schröder ${ }^{* \dagger}$}

Institute of Experimental Particle Physics, Karlsruhe Institute of Technology (KIT)

E-mail: matthias.schroeder@kit.edu

\begin{abstract}
Precise measurements of the Higgs boson couplings and cross sections provide a crucial probe of the consistency of the standard model and of hypothetical new physics effects. In this article, latest results on the Higgs boson couplings from the combination of multiple channels as well as most recent measurements of Higgs boson differential distributions by the CMS collaboration are reviewed.
\end{abstract}

European Physical Society Conference on High Energy Physics - EPS-HEP2019 -

10-17 July, 2019

Ghent, Belgium

${ }^{*}$ Speaker.
${ }^{\dagger}$ On behalf of the CMS Collaboration




\section{Introduction}

A precise measurement of the properties of the Higgs boson [1,2,3] is of paramount interest to scrutinise the standard model (SM) nature of the Higgs sector or discover new physics beyond the SM. Crucial information is obtained from investigation of the Higgs boson couplings, which can be extracted from direct measurements in individual channels or, indirectly, from the combination of measurements in multiple channels. While the coupling measurements are subject to various model assumptions, differential measurements provide a powerful, largely model-independent probe of the Higgs sector. In several channels, measurements are performed in the kinematic phase-space regions defined within the simplified template cross section (STXS) approach; in the high-resolution $\mathrm{H} \rightarrow \mathrm{ZZ}^{*} \rightarrow 4 l$ and $\mathrm{H} \rightarrow \gamma \gamma$ channels, also fully differential cross section measurements in various observables are possible.

In this article, latest measurements of the Higgs boson couplings and differential distributions by the CMS collaboration [4] are reviewed. The results have been obtained with up to $137.1 \mathrm{fb}^{-1}$ of proton-proton collision data collected during the LHC Run 2 at $13 \mathrm{TeV}$ centre-of-mass energy.

\section{Signal-Strength and Coupling Measurements}

Each combination of production and decay channel of the Higgs boson provides information on its signal strength modifier $\mu$, the measured product of cross section and branching ratio relative to the SM expectation. Combining various Higgs boson measurements with $35.9 \mathrm{fb}^{-1}$ of data collected in 2016, a global value of $\mu=1.17 \pm 0.10$ is obtained [5]. The compatibility with the SM expectation of 1 , expressed as a $p$ value, is $p=6.3 \%$.

A different parametrisation, which allows more freedom, introduces coupling modifiers $\kappa_{i}$, which modify the strength of the Higgs boson coupling to particle $i$. This provides sensitivity also to the relative sign of the couplings due to the interference between different processes contributing to the same final state. Loop-induced processes, for example Higgs boson production via the gluon-gluon fusion ( $\mathrm{ggH}$ ) mode or the decay into two photons, can be either resolved, assuming contributions from SM particles only, or effective coupling modifiers to the gluon and the photon can be introduced, which allows modelling potential new particles in the loops. Furthermore, potential decays into new particles can be considered by allowing non-SM contributions to the total Higgs boson width; in this case, degeneracies in the system are controlled by imposing the coupling to vector bosons to be $\left|\kappa_{\mathrm{V}}\right| \leq 1$. Using the 2016 data, the coupling modifiers to vector bosons and third-generation fermions can be determined with a precision of 10-20\%, and they are found to be consistent with the SM expectation [5].

These global, indirect interpretations are complemented by direct, less model-dependent measurements of each coupling strength individually. While the couplings to vector bosons have already been observed with $5 \sigma$ significance during LHC Run 1, the data collected during Run 2 allows for the first time a direct measurement of also the Higgs-fermion couplings: the couplings to the third-generation fermions have been observed in 2017 and 2018 [6, 7, 8] in agreement with the SM expectation, and the couplings to muons [9] and charm quarks [10] start to be within the experimental reach. 


\section{Simplified Template Cross Section Measurements}

The interpretation in terms of coupling modifiers is still rather model-dependent since SM Higgs boson kinematics are assumed and only the coupling strength is scaled. The STXS approach [11] is a first step towards differential, more model-independent measurements. Here, fiducial cross section measurements are performed in many exclusive phase-space regions ("bins"), defined by the Higgs boson kinematics and additional jet activity, separately per Higgs boson production channel. A common bin definition, which reflects the experimental sensitivity and theoretical relevance, is used across the experimental and theory communities, thus facilitating a straight-forward combination across channels and experiments.

The CMS collaboration has published new STXS measurements in the $\mathrm{H} \rightarrow \gamma \gamma$ and $\mathrm{H} \rightarrow \tau \tau$ channels [12, 13], obtained with $77.4 \mathrm{fb}^{-1}$ of data collected in 2016 and 2017, and in the $\mathrm{H} \rightarrow$ $\mathrm{ZZ}^{*} \rightarrow 4 l, l=e, \mu$, channel [14], using the full Run 2 dataset corresponding to $137.1 \mathrm{fb}^{-1}$.

The analyses of the $\mathrm{H} \rightarrow \gamma \gamma$ and $\mathrm{H} \rightarrow 4 l$ channels benefit from the excellent photon and lepton energy resolution, which allow reconstructing the signal as a narrow peak in the invariant di-photon and four-lepton mass spectra, respectively. The smaller branching ratio into $4 l$ is compensated by the larger signal-to-background ratio compared to the $\gamma \gamma$ final state, leading to similar sensitivity in both channels. The high resolution allows cross section measurements in finely granular kinematic regions, where the event categorisation based on reconstructed objects matches closely the corresponding STXS bins with minimal migration.

In the $\mathrm{H} \rightarrow \gamma \gamma$ channel, up to 13 bins are considered, Fig. 1 (left), which target the ggH and vector-boson fusion (VBF) production modes following the STXS stage 1 binning scheme. The sensitivity in the $\mathrm{H} \rightarrow 4 l$ channel benefits from the larger analysed dataset of the full Run 2, and cross sections have been measured in up to 22 bins of the STXS stage 1.1 scheme, targeting all primary Higgs boson production modes, Fig. 1 (right).
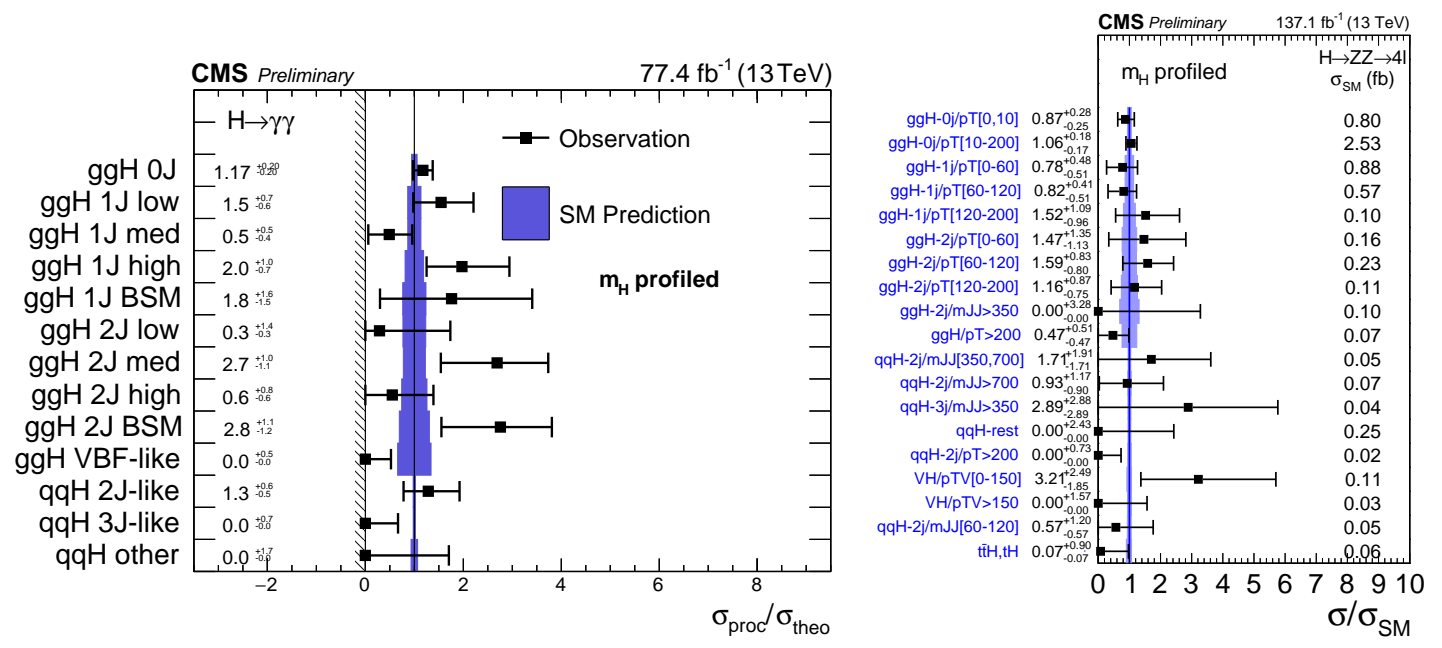

Figure 1: Ratios between the measured Higgs-boson cross sections and the SM predictions in different STXS bins in the $\mathrm{H} \rightarrow \gamma \gamma$ [12] (left) and $\mathrm{H} \rightarrow 4 l$ [14] (right) channels. The ratios are constrained to be nonnegative. The bands around the vertical lines show the theoretical uncertainty on the SM predictions. The ggH parameters include bbH components, while the qqH parameters include the hadronic VH contribution. In the $\mathrm{H} \rightarrow \gamma \gamma$ channel (left), other production modes are constrained to the SM prediction. 
In the $\mathrm{H} \rightarrow \tau \tau$ channel, four final states of the di- $\tau$ system are considered: $e \mu, e \tau_{\text {had }}, \mu \tau_{\text {had }}$, and $\tau_{\text {had }} \tau_{\text {had }}$. In order to compensate the worse resolution and signal-to-background ratio, a multiclassification neural-network is used, which combines the information provided by many different reconstructed variables. Based on the network output, the events are categorised into various background and two signal regions that target ggH and VBF production and reach categorisation efficiencies of up to $70 \%$ for VBF. In each signal category, the network output distribution provides the final discrimination between the signal and the background processes, which are determined almost entirely using data-driven methods, Fig. 2 (left). Cross sections have been measured in up to nine STXS bins of the stage 1.1 scheme, Fig. 2 (right).

In all three channels, the results are found to be compatible with the SM expectation.
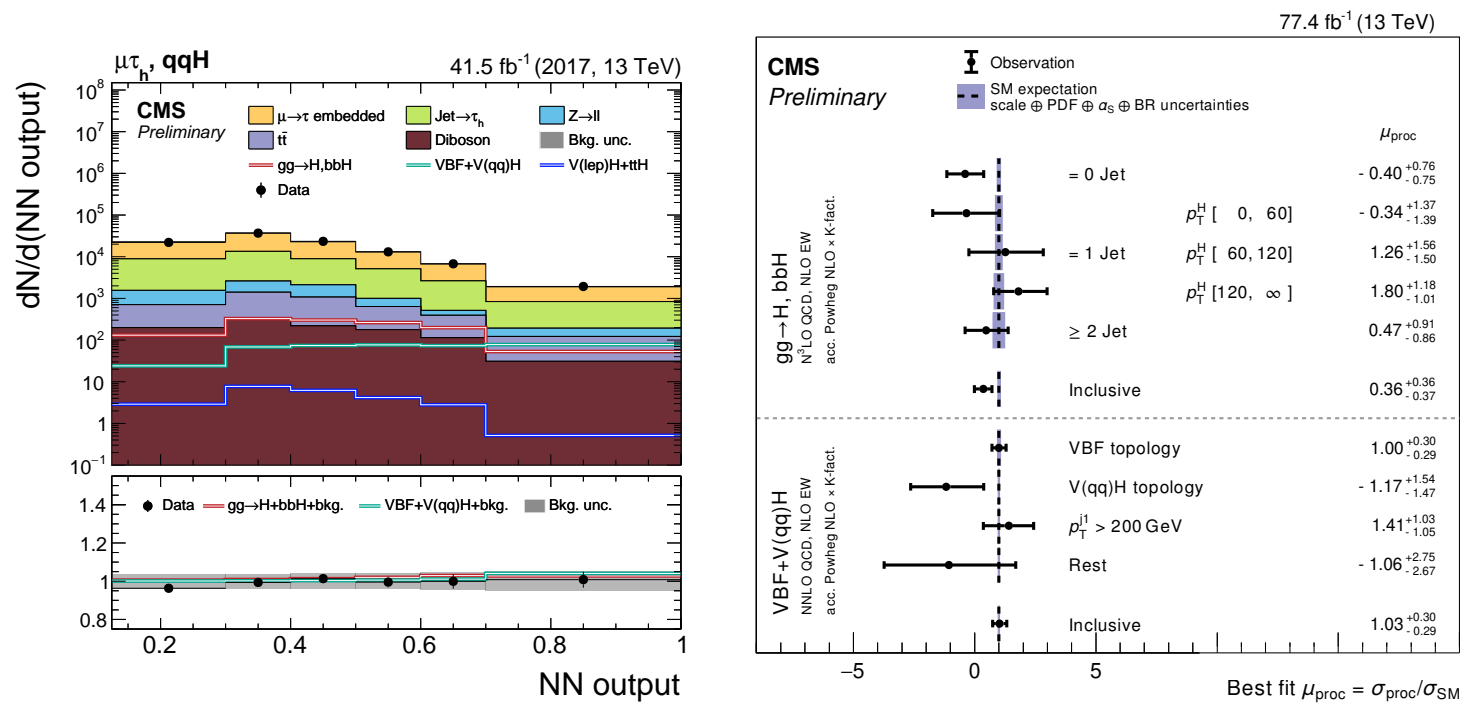

Figure 2: Neural-network output distribution in the VBF category for events in the $\mu \tau_{\text {had }}$ final state (left). The stacked histograms show the expected background and the lines the signal contributions after the fit to data. Ratios between the measured Higgs boson cross sections and the SM prediction for inclusive ggH and VBF production and in nine STXS bins (right). The bands around the vertical lines show the theoretical uncertainty on the SM predictions. [13]

\section{Fiducial Cross Section Measurements}

Beyond the STXS approach, differential cross sections in specific observables provide highly model-independent measurements and are a powerful probe of potential new physics effects. The CMS collaboration has performed differential cross section measurements mainly in the highresolution $\mathrm{H} \rightarrow 4 l$ and $\mathrm{H} \rightarrow \gamma \gamma$ channels. The cross sections are measured in fiducial phase-space regions that match closely the experimental selection, and the results are corrected to particle level via unfolding.

New results in the $\mathrm{H} \rightarrow 4 l$ channel have been obtained with the full Run 2 dataset corresponding to $137.1 \mathrm{fb}^{-1}$ [14]. Previous results in the $\mathrm{H} \rightarrow 4 l$ and the $\mathrm{H} \rightarrow \gamma \gamma$ channels [15, 16], obtained with the $35.9 \mathrm{fb}^{-1}$ of 2016 data, have been combined [17], increasing the precision by up to $30 \%$ compared to the individual measurements. At large Higgs boson $p_{\mathrm{T}}$, the precision of the combined 
result has been improved further by also taking into account results in the $\mathrm{H} \rightarrow \mathrm{b} \overline{\mathrm{b}}$ channel [18]. For the combination, the fiducial cross section measurements in each channel have been extrapolated to the full phase-space.

Cross sections have been measured as a function of various kinematic quantities of the Higgs boson and the associated jets in the event; several examples are shown in Fig. 3. The cross section as a function of the Higgs boson $p_{\mathrm{T}}$ (top row) probes the modelling of the dominant ggH production mode and provides sensitivity to potential new particles contributing to the loop. The shape of the $p_{\mathrm{T}}$ spectrum also depends on the Higgs boson couplings, and the measurement has been used to derive constraints on various couplings, among them those that are not-yet accessible directly, such as $\kappa_{c}$ [17]. The Higgs boson rapidity distribution (middle row) probes, for example, the parton distributions inside the proton, while the jet multiplicity (bottom row) is sensitive to the modelling of QCD radiation as well as the relative contributions of different Higgs boson production modes.

The presented measurements achieve a precision of typically 20-30\%, which is limited by the statistical uncertainty. The results are compatible with SM predictions computed at next-to-leading (NLO) and next-to-next-to-leading (NNLO) order perturbation theory [19, 20, 21, 22, 23, 24].

In the $\mathrm{H} \rightarrow \gamma \gamma$ channel, even double-differential cross section measurements have performed with the 2016 data, for example as a function of the Higgs boson $p_{\mathrm{T}}$ and the jet multiplicity [16]. The results are consistent with the SM expectation, but the precision is still limited by sizeable statistical uncertainties of 50-100\% or more, and significant improvements can be expected with the analysis of the full Run 2 dataset.

\section{Summary}

The CMS collaboration has performed a rich variety of Higgs boson coupling and differential measurements with the LHC Run 2 dataset. From a combined interpretation of various production and decay channels, the Higgs boson couplings to vector bosons and to third-generation fermions can be extracted at 10-20\% precision. New results in the $\mathrm{H} \rightarrow 4 l, \mathrm{H} \rightarrow \gamma \gamma$, and $\mathrm{H} \rightarrow \tau \tau$ channels provide cross section measurements in finely granular kinematic regions defined within the STXS approach. Furthermore, in the high-resolution channels $\mathrm{H} \rightarrow 4 l$ and $\mathrm{H} \rightarrow \gamma \gamma$, differential cross section measurements in various Higgs boson kinematic and event observables achieve 20-30\% precision, allowing an even more stringent test of the SM and providing a largely model-independent probe of possible new physics.

\section{Acknowledgements}

I gratefully acknowledge the financial support by the German Federal Ministry of Education and Research.

\section{References}

[1] ATLAS Collaboration, Observation of a new particle in the search for the Standard Model Higgs boson with the ATLAS detector at the LHC, Phys. Lett. B 716 (2012) 1 [1207. 7214].

[2] CMS Collaboration, Observation of a new boson at a mass of $125 \mathrm{GeV}$ with the CMS experiment at the LHC, Phys. Lett. B 716 (2012) 30 [1207. 7235]. 

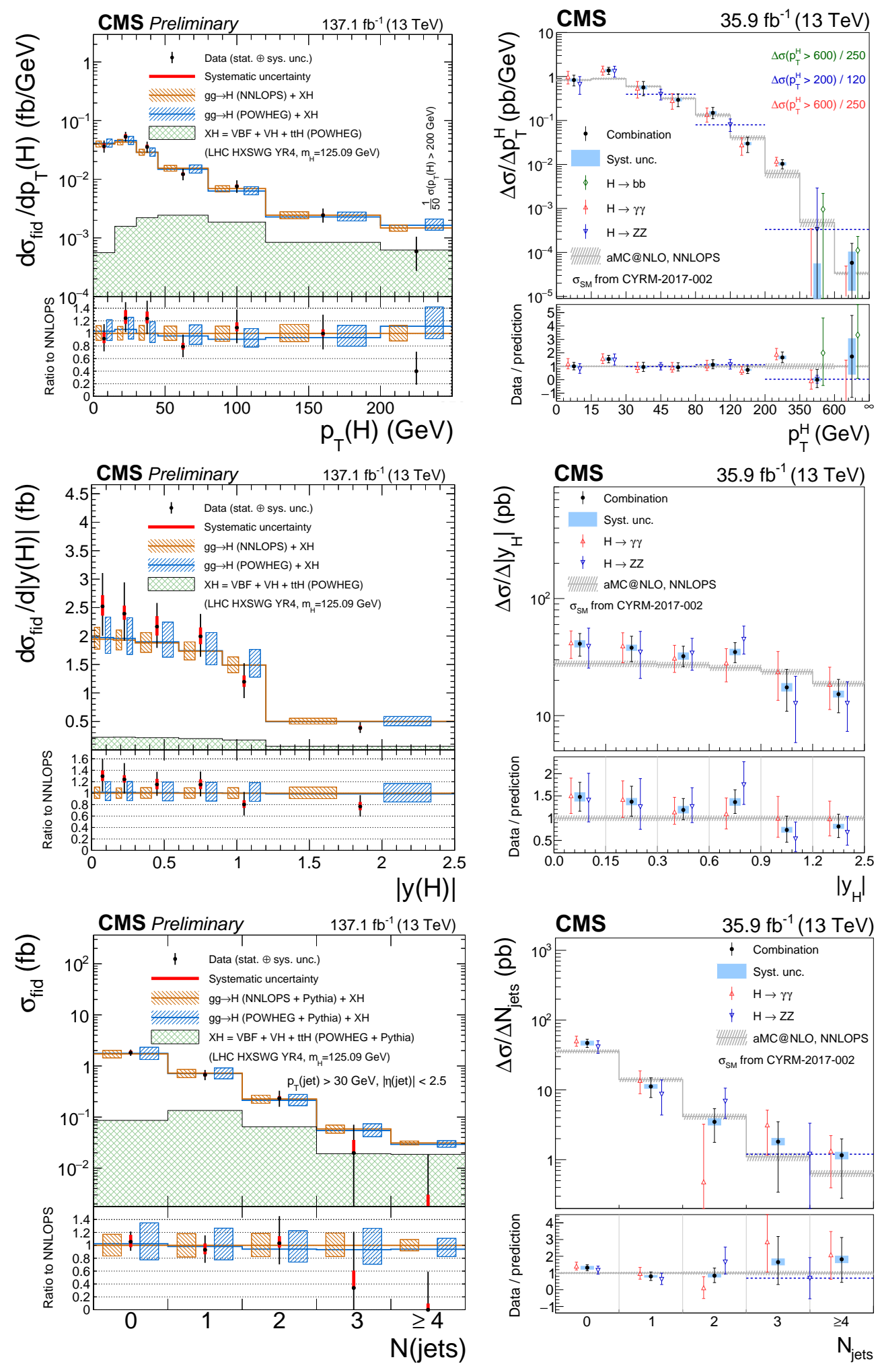

Figure 3: Fiducial differential cross sections, measured in the $4 l$ channel with $137.1 \mathrm{fb}^{-1}$ [14] (left), and total differential cross section, obtained from the combination of measurements in the $4 l, \gamma \gamma$, and b $\bar{b}$ channels with $35.9 \mathrm{fb}^{-1}$ [17] (right) for the Higgs boson $p_{\mathrm{T}}$ (top) and rapidity (middle), and for the jet multiplicity (bottom). The rightmost bins include overflow contributions. 
[3] CMS Collaboration, A new boson with a mass of $125 \mathrm{GeV}$ observed with the CMS experiment at the Large Hadron Collider, Science 338 (2012) 1569.

[4] CMS Collaboration, The CMS experiment at the CERN LHC, JINST 3 (2008) S08004.

[5] CMS Collaboration, Combined measurements of Higgs boson couplings in proton-proton collisions at $\sqrt{s}=13 \mathrm{TeV}$, Eur. Phys. J. C79 (2019) 421 [1809.10733].

[6] CMS Collaboration, Observation of the Higgs boson decay to a pair of $\tau$ leptons with the CMS detector, Phys. Lett. B779 (2018) 283 [1708.00373].

[7] CMS Collaboration, Observation of t $\bar{t} H$ production, Phys. Rev. Lett. 120 (2018) 231801 [1804.02610].

[8] CMS Collaboration, Observation of Higgs boson decay to bottom quarks, Phys. Rev. Lett. 121 (2018) 121801 [1808.08242].

[9] CMS Collaboration, Search for the Higgs boson decaying to two muons in proton-proton collisions at $\sqrt{s}=13$ TeV, Phys. Rev. Lett. 122 (2019) 021801 [1807.06325].

[10] CMS Collaboration, Search for the standard model Higgs boson decaying to charm quarks, CMS Physics Analysis Summary CMS-PAS-HIG-18-031, CERN, 2019.

[11] LHC Higgs Cross Section Working Group, Handbook of LHC Higgs Cross Sections: 4. Deciphering the Nature of the Higgs Sector, 1610.07922.

[12] CMS Collaboration, Measurements of Higgs boson production via gluon fusion and vector boson fusion in the diphoton decay channel at $\sqrt{s}=13 \mathrm{TeV}$, CMS Physics Analysis Summary CMS-PAS-HIG-18-029, CERN, 2019.

[13] CMS Collaboration, Measurement of Higgs boson production and decay to the $\tau \tau$ final state, CMS Physics Analysis Summary CMS-PAS-HIG-18-032, CERN, 2019.

[14] CMS Collaboration, Measurements of properties of the Higgs boson in the four-lepton final state in proton-proton collisions at $\sqrt{s}=13 \mathrm{TeV}$, CMS Physics Analysis Summary CMS-PAS-HIG-19-001, CERN, 2019.

[15] CMS Collaboration, Measurements of properties of the Higgs boson decaying into the four-lepton final state in pp collisions at $\sqrt{s}=13 \mathrm{TeV}$, JHEP 11 (2017) 047 [1706.09936].

[16] CMS Collaboration, Measurement of inclusive and differential Higgs boson production cross sections in the diphoton decay channel in proton-proton collisions at $\sqrt{s}=13 \mathrm{TeV}$, JHEP 01 (2019) 183 [1807.03825].

[17] CMS Collaboration, Measurement and interpretation of differential cross sections for Higgs boson production at $\sqrt{s}=13 \mathrm{TeV}$, Phys. Lett. B792 (2019) 369 [1812.06504].

[18] CMS Collaboration, Inclusive search for a highly boosted Higgs boson decaying to a bottom quark-antiquark pair, Phys. Rev. Lett. 120 (2018) 071802 [1709. 05543 ].

[19] M. Grazzini and H. Sargsyan, Heavy-quark mass effects in Higgs boson production at the LHC, JHEP 09 (2013) 129 [1306.4581].

[20] D. de Florian, G. Ferrera, M. Grazzini and D. Tommasini, Higgs boson production at the LHC: transverse momentum resummation effects in the $H->2$ gamma, $H->W W->\ln u \ln u$ and $H->Z Z->4 l$ decay modes, JHEP 06 (2012) 132 [1203.6321].

[21] E. Bagnaschi, G. Degrassi, P. Slavich and A. Vicini, Higgs production via gluon fusion in the POWHEG approach in the SM and in the MSSM, JHEP 02 (2012) 088 [1111.2854]. 
[22] K. Hamilton, P. Nason, E. Re and G. Zanderighi, NNLOPS simulation of Higgs boson production, JHEP 10 (2013) 222 [1309. 0017$].$

[23] K. Hamilton, P. Nason and G. Zanderighi, MINLO: Multi-Scale Improved NLO, JHEP 10 (2012) 155 [1206.3572].

[24] A. Kardos, P. Nason and C. Oleari, Three-jet production in POWHEG, JHEP 04 (2014) 043 [1402.4001]. 